\title{
Caractérisation physico-chimique et sensorielle de diverses formulations de jus à base de pomme de cajou et d'ananas
}

\author{
Gninfanni Silvère OUATTARA ${ }^{1 *}$, Doudjo $\mathrm{SORO}^{2}$, Kouamé Olivier CHATIGRE ${ }^{1}$ et \\ Ernest Kouadio KOFFI ${ }^{1}$
}

\author{
${ }^{1}$ Laboratoire de Biochimie et Sciences des Aliments, UFR Biosciences, Université Félix Houphouët-Boigny, \\ UFHB 01 BP V 34 Abidjan, Côte d'Ivoire. \\ ${ }^{2}$ UMRI Sciences des Procédés Alimentaires, chimiques et Environnementaux, Institut National Polytechnique \\ Félix Houphouët-Boigny (INP-HB), BP 1313 Yamoussoukro, Côte d'Ivoire. \\ *Auteur correspondant; E-mail : silveroouatt@gmail.com; Tel : (225) 49021286.
}

\section{RESUME}

En Côte d'Ivoire, la quasi-totalité des pommes de cajou ne sont pas transformées à cause de leur astringence et de certains tabous. Pour y remédier, cette étude vise à formuler quatre boissons non alcoolisées à base de jus de pomme de cajou et d'ananas. Les différentes formulations ont été soumises à l'appréciation d'un jury de dégustateurs puis à des analyses physico-chimiques à l'aide de méthodes normées. Les dégustateurs ont préféré la formulation $\mathrm{F} 4$ contenant $25 \%$ de jus de pomme de cajou et $75 \%$ de jus d'ananas. Ce cocktail est sucré $\left(11,5^{\circ}\right.$ brix $)$, moins astringent par rapport aux autres formulations et a une teneur en phénols totaux de 0,6 $\mathrm{mg} / \mathrm{l} \mathrm{Eq} \mathrm{Ac.} \mathrm{Gallique.} \mathrm{La} \mathrm{teneur} \mathrm{en} \mathrm{vitamine} \mathrm{C}$ du jus brut de cajou est de 317,5 mg/100 ml tandis que la formulation F4 contient 52,03 $\mathrm{mg} / 100 \mathrm{ml}$ de vitamine $\mathrm{C}$. La teneur en cendres est plus élevée dans la formulation F4 (0,41\%). On note aussi la présence d'un nombre plus important de composés osidiques. (C) 2016 International Formulae Group. All rights reserved.

Mots clés : Boissons, ananas, pomme de cajou, analyses physico-chimiques.

\section{Physicochemical and sensory characterization of various formulations of cashew apple juice and pineapple}

\begin{abstract}
In Ivory Coast, almost all cashew apples are not processed because of their astringency and taboos. In response, this study aims to make four soft drinks from cashew apple juice and pineapple. The different formulations were submitted to a jury of tasters then physicochemical analyzes using standardized methods were conducted. The tasters preferred the F4 formulation containing $25 \%$ of cashew apple juice and $75 \%$ of pineapple juice. This cocktail is sweeter (11.5 $\left.{ }^{\circ} \mathrm{brix}\right)$, less astringent compared to other formulations and has a total phenol content of $0.6 \mathrm{mg} / 1 \mathrm{Ac}$ Eq. Gallic. The vitamin C content of raw cashew juice is $317.5 \mathrm{mg} / 100 \mathrm{ml}$ while formulation F4 contains $52.03 \mathrm{mg} / 100 \mathrm{ml}$ of vitamin C. The ash content is higher in formulation F4 $(0$, $41 \%$ ). A larger number of saccharides were found in the preferred formulation.

(C) 2016 International Formulae Group. All rights reserved.
\end{abstract}


Keywords: Drinks, pineapple, cashew apple, physicochemical analyses.

\section{INTRODUCTION}

La production de noix de cajou en Côte d'Ivoire a subi un accroissement notable passant de 6000 tonnes en 1990 à 725000 tonnes en 2015 (CCA, 2016), faisant du pays le premier producteur mondial de noix de cajou brutes. La production mondiale quant à elle a presque doublée en moins d'une décennie, passant de 2.361.384 tonnes en 2002 à 4.152 .315 tonnes en 2012 (FAO, 2014).

Le fruit de l'anacardier est composé de deux parties : la noix et la pomme. La noix de cajou (vrai fruit de l'anacardier) est constituée d'une amande et d'une coque qui contient le baume de cajou ou CNSL (Cashew Nut Shell Liquid). La pomme, quant à elle, est le pédoncule hypertrophié qui relie l'arbre à la noix. Le tout tombe de l'arbre une fois à maturité. La maturation progressive impose de renouveler la récolte tous les 2 à 4 jours pour les pommes de cajou (Lautié et al., 2001). La culture de l'anacardier contribue au développement socio-économique de plusieurs pays dans le monde (Aboh et al., 2011). Ce développement socio-économique serait plus notable si la pomme de cajou qui représente 9 à 10 fois le poids de la noix, perdue généralement sur les lieux de récolte était transformée industriellement.

En effet les pommes de cajou ne sont pas transformées en Côte d'Ivoire notamment à cause de leur astringence et de certains tabous (Soro et al., 2008). La pomme de cajou est très riche en éléments nutritifs. Elle est constituée de $85 \%$ d'un jus sucré, acide, riche en vitamine $\mathrm{C}$ ( 6 fois plus riche que l'orange douce), en caroténoïdes (Assunção et Mercadante, 2003) et en polyphénols (Michodjehoun-Mestres et al., 2009). Les polyphénols réduisent l'oxydation des LDL et VLDL (Vinson et al., 2001). Ainsi ils jouent un rôle très important dans la prévention des maladies cardio-vasculaires et d'autres pathologies chroniques. Le jus de pomme de cajou pourrait donc être une boisson nutritive pour les populations rurales et citadines.

L'Institut de Recherche pour le Développement (IRD) a recensé et documenté dans le bassin du fleuve Gambie, en Casamance et en Guinée Bissau, près d'une cinquantaine de recettes culinaires à base de pomme de cajou (IRD, 2012). Ainsi la pomme de cajou peut-être transformée en jus, en confiture, en vin, en vinaigre et en alcool (Dedehou et al., 2015).

$\mathrm{La}$ principale voie de valorisation envisagée pour la pomme de cajou reste sa transformation en jus. Mais celle-ci se heurte à diverses difficultés telles que l'astringence du jus liée à la forte concentration de tannins condensés (Soro, 2008), la grande thermosensibilité du produit aussi bien en terme nutritionnel que sensoriel et sa richesse en sucres réducteurs responsables de la réaction de Maillard lors des traitements thermiques (Soro, 2008).

La formulation d'un cocktail non alcoolisé avec un fruit tropical non astringent pourrait non seulement permettre une valorisation de la pomme de cajou mais aussi une amélioration de l'acceptabilité de ce jus. Pour ce faire, l'ananas (Ananas comosus), pourrait être utilisé car c'est un fruit apprécié par la population et de teneur en vitamine $\mathrm{C}$ relativement basse, environ $14,70 \mathrm{mg} / 100 \mathrm{ml}$ selon Akinwale (2000). En outre c'est un fruit non astringent et moins acide à maturité. De plus, à l'état frais, l'ananas contient plusieurs enzymes dont la broméline qui facilite la digestion et l'assimilation des protéines, en fractionnant les chaines d'acides aminés (Sekhar et al., 2013).

L'objectif de cette étude est de formuler un cocktail à base de jus de pomme de cajou et de jus d'ananas de bonne qualité nutritive et sensorielle. 


\section{MATERIEL ET METHODES Matériel biologique}

Le matériel végétal utilisé était constitué de pommes de cajou (Anacardium occidentale L. Anacardiaceae) et d'ananas (Ananas comosus). Les pommes de cajou (Figure 1) utilisées sont composées de pommes rouges et jaunes provenant du centre de la Côte d'Ivoire dans la région des lacs plus précisément de la ville de Yamoussoukro. L'ananas (Figure 2) utilisé est de variété Cayenne lisse au stade mûr et provient de Bonoua, ville située au Sud de la Côte d'Ivoire.

\section{Méthode de production du jus de pomme de cajou}

Les fruits récoltés sont constitués de pommes et de noix de cajou. Les pommes ont été soigneusement séparées des noix à l'aide d'un fil attaché aux deux extrémités par des petits morceaux de bois pour ne pas les endommager. Les pommes obtenues ont été trempées dans de l'eau javellisée à 100 ppm de chlore actif pendant 20 minutes puis rincées à l'eau claire. Après le rinçage et le broyage, l'extraction du jus a été réalisée à l'aide d'une presse à pulpe de marque ZBK220077-88LW74d (B) A. Les différentes opérations unitaires sont présentées par la Figure 3. Le jus a été ensuite filtré à l'aide d'un tamis de mailles de $1 \mathrm{~mm}$ de diamètre puis conditionné dans des bidons de 5 litres et conservé à $-40{ }^{\circ} \mathrm{C}$ avant la formulation du cocktail.

\section{Méthode de production du jus d'ananas}

Les fruits récoltés ont été trempés dans de l'eau javellisée à $100 \mathrm{ppm}$ de chlore actif pendant 20 minutes puis rincé à l'eau claire. Après le lavage, les ananas ont été épluchés pour être séparés de leurs ensilages, épluchures et axes centraux. Les ananas ont été découpés puis broyés dans un mixeur (Kenwood blender). Après le broyage le jus a été filtré à l'aide d'un tamis de mailles de 1 $\mathrm{mm}$ de diamètre dans des conditions aseptiques, puis conditionné dans des bidons de 5 litres et conservé en chambre froide à $40{ }^{\circ} \mathrm{C}$ avant les différentes formulations de cocktail. Les différentes opérations unitaires sont présentées par la Figure 4.

\section{Méthode de formulation du jus à base de} pomme de cajou et d'Ananas

Quatre formulations ont été réalisées:

La formulation $\mathrm{F} 1$, constituée de $100 \%$ de jus de pomme de cajou; la formulation F2 qui est un mélange de $75 \%$ de jus de pomme de cajou et $25 \%$ de jus d'ananas; la formulation $\mathrm{F} 3$, composée de $50 \%$ de jus de pomme de cajou et $50 \%$ de jus d'ananas et enfin la formulation $\mathrm{F} 4$, constituée de $25 \%$ de jus pomme de cajou et $75 \%$ de jus d'ananas.

\section{Analyse sensorielle}

L'analyse sensorielle a consisté à la réalisation de deux tests à savoir le test de préférence par classement et le test descriptif.

\section{Test de préférence par classement.}

Le panel a été constitué avec 100 personnes non entrainées, recrutées sur la base de leur disponibilité et n'ayant pas d'aversion pour le produit. Les échantillons de jus et de cocktails de jus ont été présentés simultanément et de manière randomisé à chaque panéliste suivant une distribution en aveugle (échantillons codés). Chaque panéliste a classé les différentes formulations en fonction du plaisir ressenti. A la suite du test de préférence par classement, la boisson classée première a été retenue puis un test descriptif a été réalisé sur celle-ci.

\section{Analyse descriptive}

La méthode a consisté à évaluer et quantifier les descripteurs appropriés (couleur, fluidité, clarté, arôme, saveur et astringence) selon une échelle de catégorie. L'intensité des descripteurs a été évaluée sur une échelle non structurée de $10 \mathrm{~cm}$. De 0 pour descripteur non perçu à 10 pour extrêmement intense. Les échantillons ont été présentés à un panel composé de 15 juges. Ces derniers sont composés de femmes et d'hommes. Les juges ont été sélectionnés sur la base de leur disponibilité et n'ayant pas d'aversion pour le produit. Leur acuité sensorielle, leur motivation et leur capacité à décrire un 
produit ont été aussi des critères de sélection. Ce jury a été entrainé à l'analyse sensorielle. Les échantillons ont été codés (avec trois chiffres) et présentés de façon monadique à chaque panéliste.

\section{Analyse statistique}

L'analyse statistique a été réalisée par le logiciel Statistica 7.1. Les comparaisons entre les variables ont été déterminées par analyse de variance ANOVA, test de Student Newman Keuls dont le seuil de significativité est de 5\%. Les données de l'analyse sensorielle ont été soumises à une analyse de variance (ANOVA) à effet principaux suivi du test de séparation des moyennes de Honestly Significant Différence (HSD) de Tukey à $\mathrm{P}<$ $1 \%$.

\section{Analyses physico-chimiques}

Le $\mathrm{pH}$ a été déterminé selon la méthode de l'AOAC (1995), $50 \mathrm{~mL}$ de jus ont servi à la détermination $\mathrm{du} \mathrm{pH}$ après étalonnage $\mathrm{du} \mathrm{pH}$ mètre (Model CYBERSCAN PH 11).

L'acidité titrable a été déterminée par dosage acido-basique avec une solution de soude à $0,1 \mathrm{~N}$, en présence de phénolphtaléine comme indicateur coloré selon la méthode de l'AOAC (1995).

L'extrait sec réfractométrique a été déterminé par la méthode de l' AOAC (1995). Une goutte du cocktail de jus a été déposée sur le verre du réfractomètre à poche (Model ATAGO POCKET REFRACTOMETER) pour en évaluer la quantité de matières en suspension. La lecture de la valeur de l'extrait $\mathrm{sec}$ réfractométrique a été faite à la lumière, au niveau de l'oculaire de l'appareil.

La teneur en acide ascorbique est mesurée par la méthode de titration au 2,6dichlorophénol-indophénol (2,6-DCPIP). La méthode consiste en une réduction du 2,6DCPIP et en une oxydation de l'acide ascorbique en acide déhydroascorbique. Elle permet donc une détermination de la teneur en vitamine $\mathrm{C}$ sous sa forme réduite (acide ascorbique). Une fois que l'oxydation de l'acide ascorbique est terminée, le 2,6-DCPIP sert d'indicateur coloré et sa présence en solution donne une couleur rose caractéristique (AOAC, 1984).

Le taux de matière sèche a été déterminé selon la méthode par étuvage (AOAC, 1990). Un creuset de masse Mo contenant l'échantillon de masse $\mathrm{M}_{1}(10 \mathrm{~g})$ a été placé à l'étuve à $105{ }^{\circ} \mathrm{C}$. Après trois heures, le creuset a été refroidi au dessiccateur pour l'empêcher de prendre de l'humidité avant d'être pesé et remis à nouveau à l'étuve. Après 30 minutes, la même opération a été effectuée. Elle a été répétée jusqu'à l'obtention des masses $\mathrm{M}_{2}$ sensiblement identiques avec un écart de $0,05 \mathrm{~g}$.

La teneur en protéine a été déterminée selon la méthode AOAC (1990). L'azote total a été dosé selon la méthode de Kjeldahl après minéralisation sulfurique en présence de catalyseur au sélénium. La teneur en azote a été multipliée par 6,25 (coefficient de conversion de l'azote en protéines).

Les lipides ont été extraits par l'hexane dans un extracteur de type Soxhlet (Unid Tecator, System HT2 1045, Suède). Après évaporation du solvant et séchage de la capsule à l'étuve à $105{ }^{\circ} \mathrm{C}$ pendant $30 \mathrm{mn}$; la différence de poids a donné la teneur en lipides de l'échantillon (AOAC, 1990).

Les taux de cendres des cocktails ont été déterminés selon la méthode AOAC (1995). Elle a consisté à calciner cinq grammes de cocktail de jus dans un four à moufle oxydante à $550{ }^{\circ} \mathrm{C}$ pendant 24 heures.

La méthode de Wood et al. (2002) a été utilisée pour le dosage des polyphénols totaux. Un volume de $2,5 \mathrm{ml}$ de réactif de FolinCiocalteu dilué $(1 / 10)$ a été ajouté à $30 \mu \mathrm{l}$ d'extrait. Le mélange a été maintenu pendant 2 minutes dans l'obscurité à température ambiante, puis $2 \mathrm{ml}$ de solution de carbonate de calcium ( $75 \mathrm{~g} / \mathrm{l})$ ont été ajoutés. Ensuite, le mélange a été placé pendant 15 minutes au bain-marie à $50{ }^{\circ} \mathrm{C}$, puis refroidi rapidement.

L'absorbance a été mesurée à $760 \mathrm{~nm}$, avec de l'eau distillée comme blanc. Une droite d'étalonnage a été réalisée avec l'acide gallique à différentes concentrations.

Les différents sucres présents dans les échantillons de jus ont été identifiés à l'aide d'un chromatographe en phase gazeuse (HP 
$6890 \mathrm{~N})$ de waters, couplé à un Spectromètre de masse tandem (Quattro micro GC) de Micromass. Les échantillons ont été injectés à la dose de 1 microlitre avec une tranche de 1:10 microlitre. Le réactif de dérivation est le BSTFA $+1 \%$ TMCS. Les sucres ont été dosés après formation de leurs dérivés triméthylsilylés.

\section{RESULTATS}

\section{Analyse sensorielle}

\section{Test de classement préférentiel}

Les résultats du test de classement préférentiel présentés dans le Tableau 1 montrent que les boissons formulées ont été diversement appréciées. La formulation la plus appréciée est la F4 (25\% du jus de pomme de cajou et $75 \%$ du jus d'ananas), ensuite vient la F3 (50\% du jus de pomme de cajou et $50 \%$ du jus d'ananas), la F2 (75\% du jus de pomme de cajou et $25 \%$ du jus d'ananas) et enfin la F1 (100\% du jus de pomme de cajou).

\section{Test descriptif}

La Figure 5 laisse apparaitre des caractéristiques sensorielles moyennes du jus de pomme de cajou et de la boisson F4 (25\% de jus de pomme de cajou et $75 \%$ de jus d'ananas). Les caractéristiques étudiées sont : la couleur, la fluidité, la clarté, l'arôme, la saveur et l'astringence.

Tous les jus sont de couleur jaune. La formulation F4 a une couleur jaune plus foncée que le jus brut de pomme de cajou. Cette formulation a un arôme ananas beaucoup plus prononcé. La fluidité et la clarté des deux formulations sont sensiblement voisines. La formulation F4 est plus sucrée $(8,9 \mathrm{~cm})$ que le jus brut de pomme de cajou $(8 \mathrm{~cm})$. Cette formulation est moins acide $(3 \mathrm{~cm})$ et moins astringente $(2 \mathrm{~cm})$ que le pur jus de pomme de cajou dont l'acidité est de 5,4 cm et l'astringence de $6,2 \mathrm{~cm}$. Ces deux formulations ne sont ni amer ni salée.

\section{Caractéristiques physico-chimiques}

Les résultats du Tableau 2 rendent compte de la composition physico-chimique des différentes formulations de boissons. La teneur en polyphénols totaux est significativement très élevée $(1,8 \mathrm{mg} / \mathrm{ml} \mathrm{Eq}$ Ac. Gallique) au niveau du jus brut de pomme de cajou $(\mathrm{p}<0,05)$ tandis que le taux le plus bas $(0,6 \mathrm{mg} / \mathrm{ml} \mathrm{Eq} \mathrm{Ac.} \mathrm{Gallique)} \mathrm{est} \mathrm{contenu}$ dans la formulation F4 qui est la préférée des dégustateurs.

La teneur en vitamine $C$ est plus élevée $(317,5 \mathrm{mg} / 100 \mathrm{ml})$ dans la formulation $\mathrm{F} 1$ (contenant $100 \%$ du jus de pomme de cajou) et moins élevée $(52,03 \mathrm{mg} / 100 \mathrm{ml})$ dans la formulation $\mathrm{F} 4$ ( $25 \%$ de jus de pomme de cajou et $75 \%$ de jus d'ananas). La formulation F3 est le cocktail le plus acide avec un $\mathrm{pH}$ de 3,53 tandis que la formulation $\mathrm{F} 4$ possède le pH le plus élevé $(4,52)$. L'extrait sec réfractométrique de la formulation $\mathrm{F} 4(11,5$ ${ }^{\circ}$ brix) est plus élevé que celui de la formulation F1 ( $7^{\circ}$ brix). La teneur en cendres est significativement élevée dans la formulation F4 $(0,41 \%)$ et plus basse dans la F2 (0,21\%).

Les résultats de l'analyse qualitative des sucres du jus brut de pomme de cajou sont présentés par la Figure 4. Plusieurs oses et dérivés ont été identifiés: le DErythrotetrotetrofuranose ; le DXylopyranose; le 2-Deoxy-galactopyranose ; le D-Fructose; le Glucose, $\alpha$-methyl; le DGlucose; le 6-Deoxy-L-Mannose; $\alpha$-DXylopyranose; le D-Mannose; le DGlucosamine; le Cis-Inositol; le D-Threitol et le Melibiose.

La Figure 5 rend compte de la composition qualitative des sucres de la formulation F4 préférée par les dégustateurs. Plusieurs oses et dérivés ont été identifiés parmi lesquels:

le Methyl 3-o-acetyl-2,4-di-o-methyl- $\alpha$ D-Xylopyranoside; le D-Glucosamine; le cis-Inositol; le Methyl (methyl 3-o-acetyl2,4-di-o-methyl- $\alpha$-D-Mannopyranoside) urinate ; le D-Glucose; le D-Galactose; le DGluco-hexodialdofuranoside ; le 3-Tosyl sedoheptulose; le D-Threitol; le DRibofuranose; le $\beta$-D-Glucopyranose et le Melibiose. 


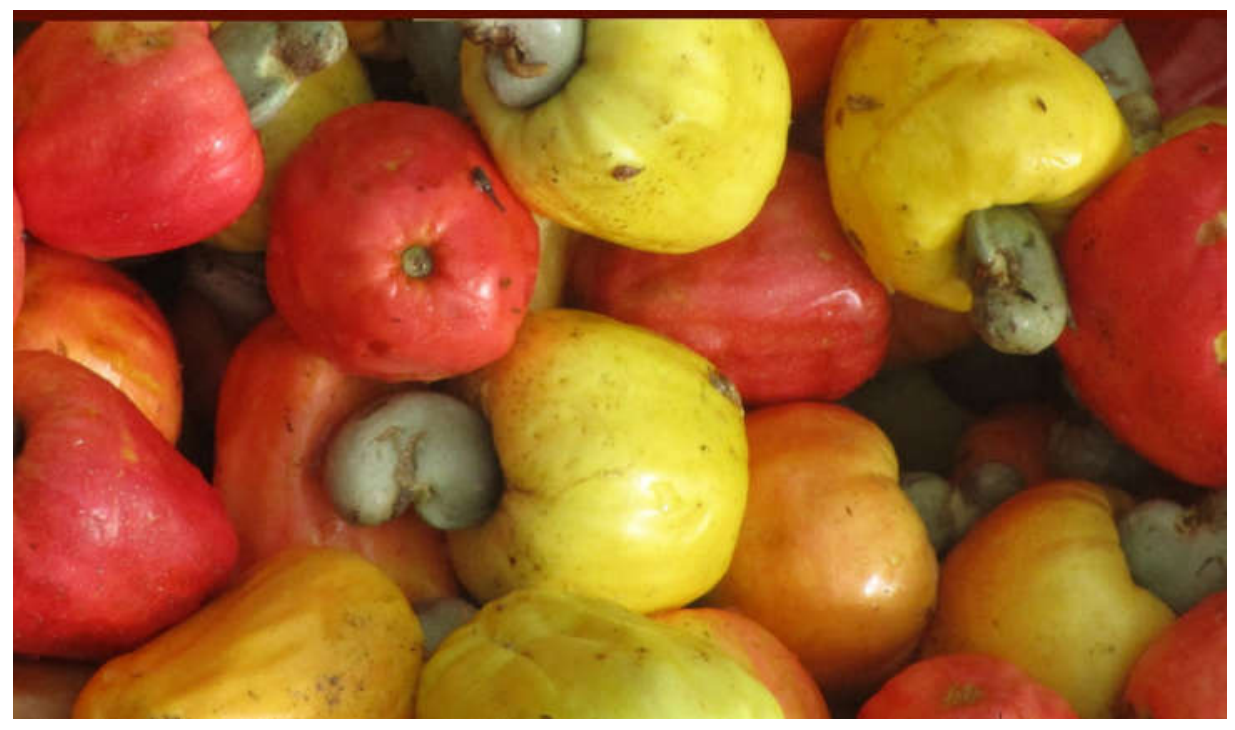

Figure 1 : Pomme de cajou.

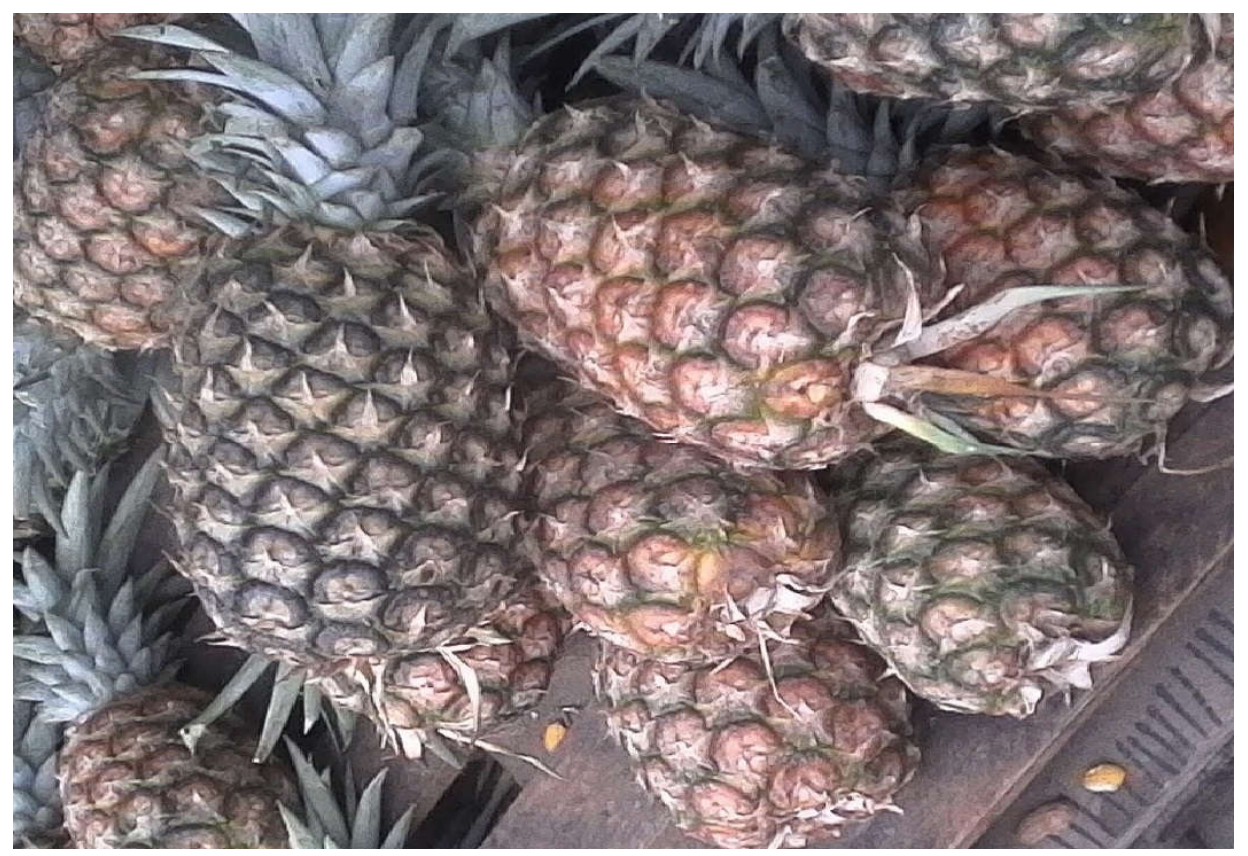

Figure 2 : Ananas. 


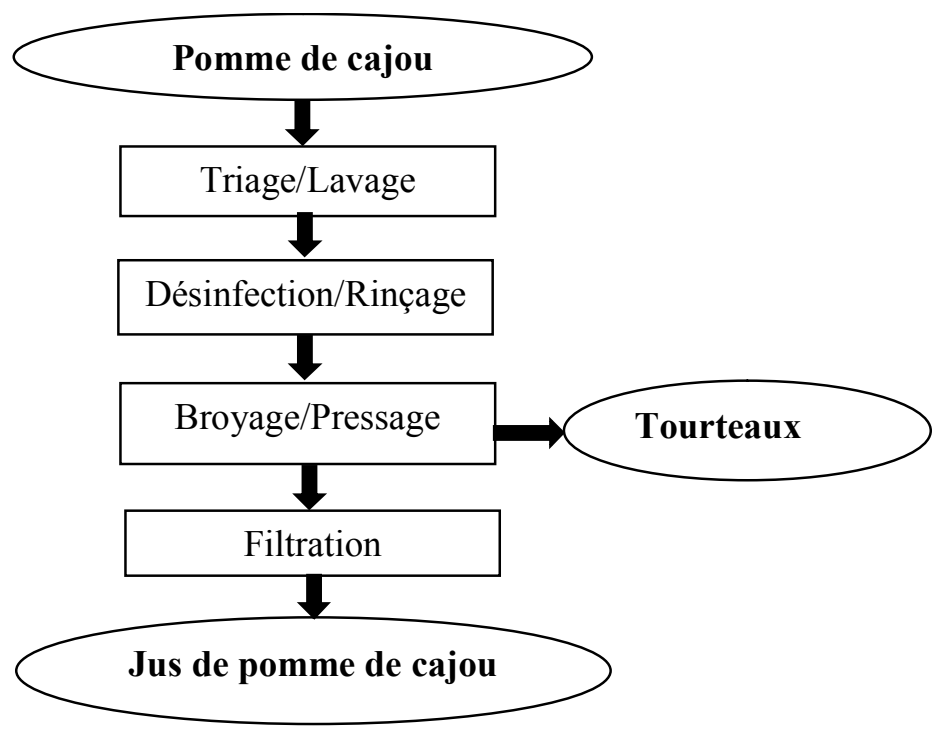

Figure 3 : Diagramme de production de jus de pomme de cajou

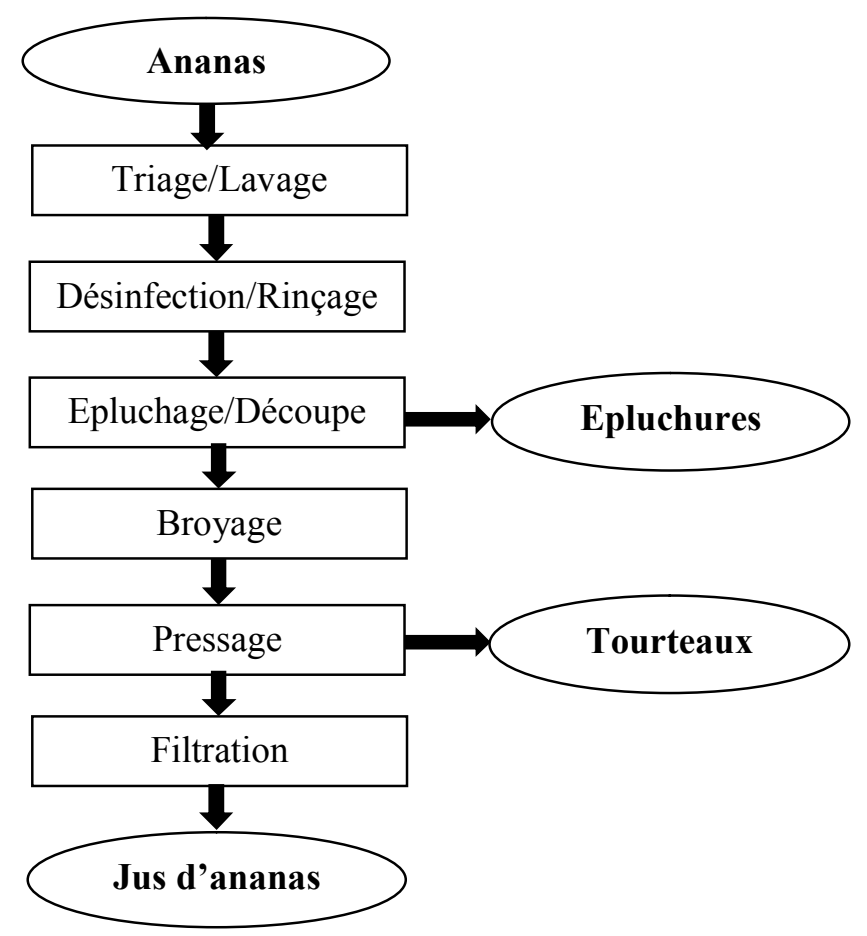

Figure 4 : Diagramme de production de jus d'ananas 


\section{- Formulation F4 (25\% de jus de pomme de cajou et $75 \%$ de jus d'ananas) - Formulation F1 (100\% de jus de pomme de cajou)}

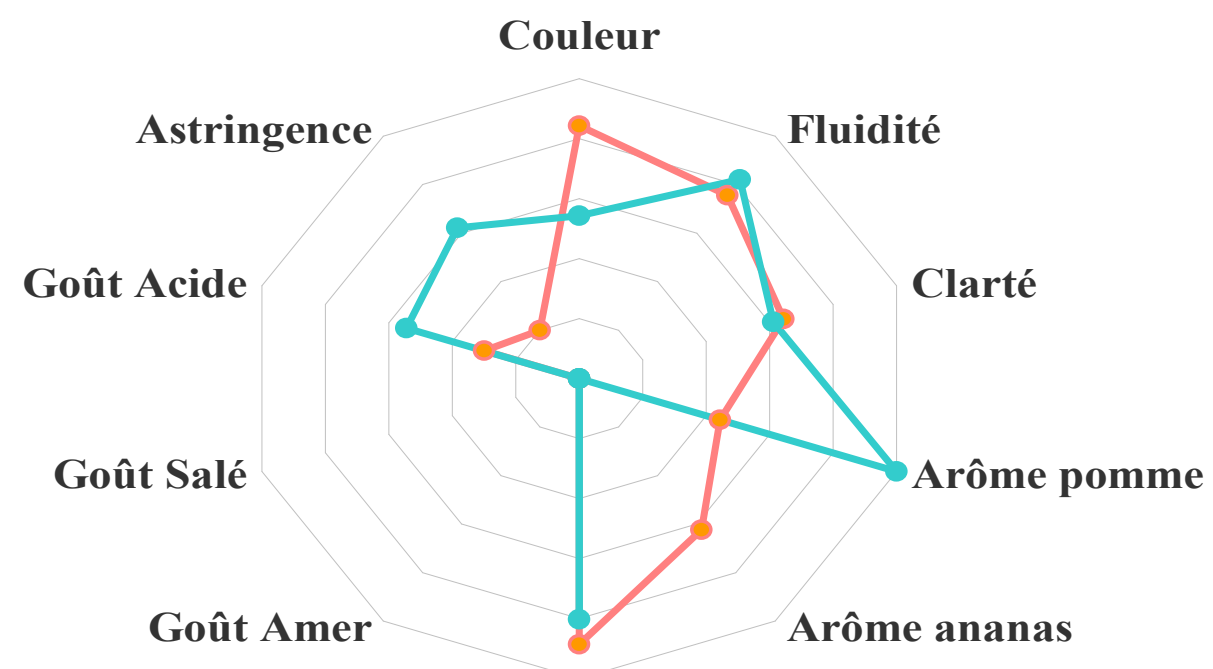

\section{Goût sucré}

Figure 5 : Profil sensoriel de la formulation F1 (100\% jus de pomme de cajou) et F4 ( $25 \%$ de jus de pomme de cajou et $75 \%$ de jus d'ananas.

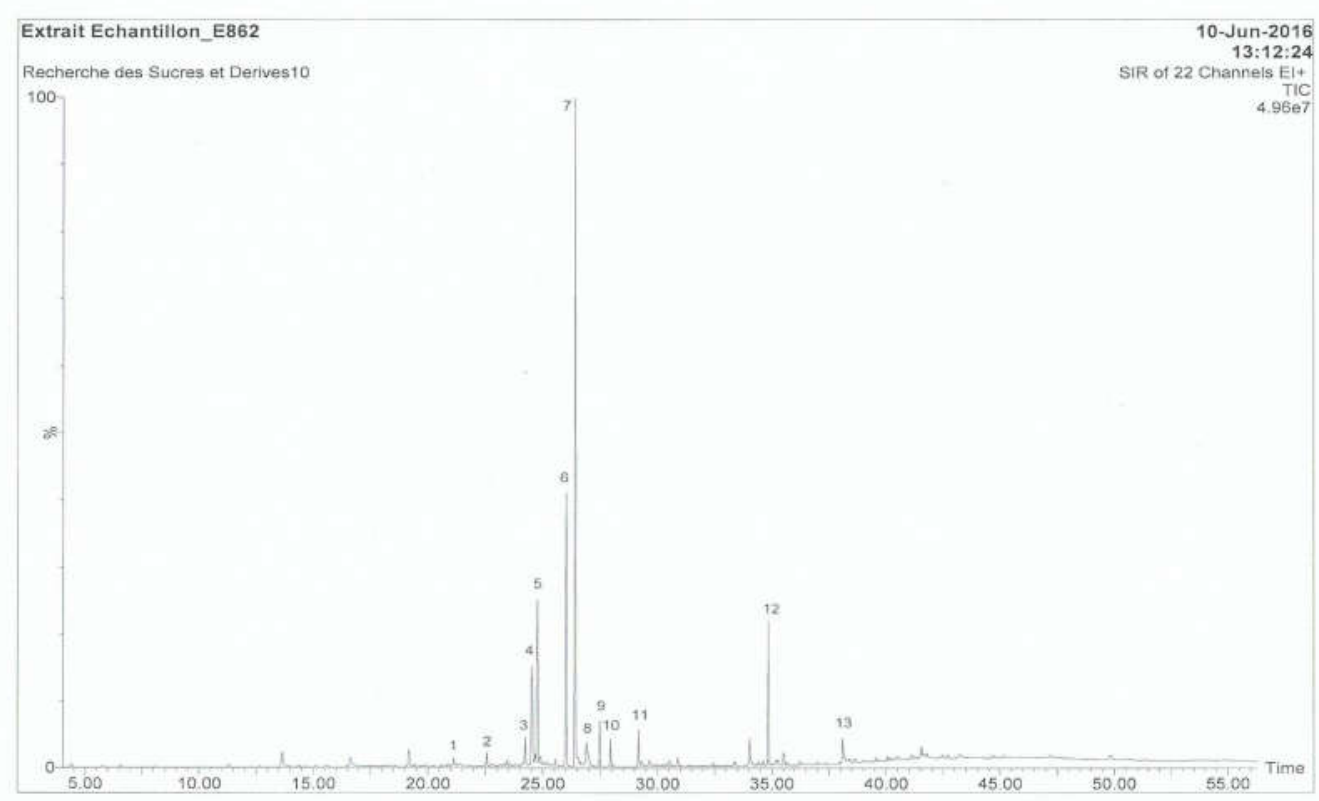

Figure 6 : Chromatogramme GC-MS de jus de pomme de cajou.

1.le D-Erythrotetrotetrofuranose, 2. le D- Xylopyranose ; 3. le 2-Deoxy-galactopyranose ; 4. le D-Fructose ; 5 . le Glucose, $\alpha$ methyl; 6 .

Le D-Glucose; 7. le 6-Deoxy-L-Mannose; 8. $\alpha$-D-Xylopyranose; 9. le D-Mannose; 10. le D-Glucosamine; 11. le Cis-Inositol; 12. le D-Threitol; 13.le Melibiose. 


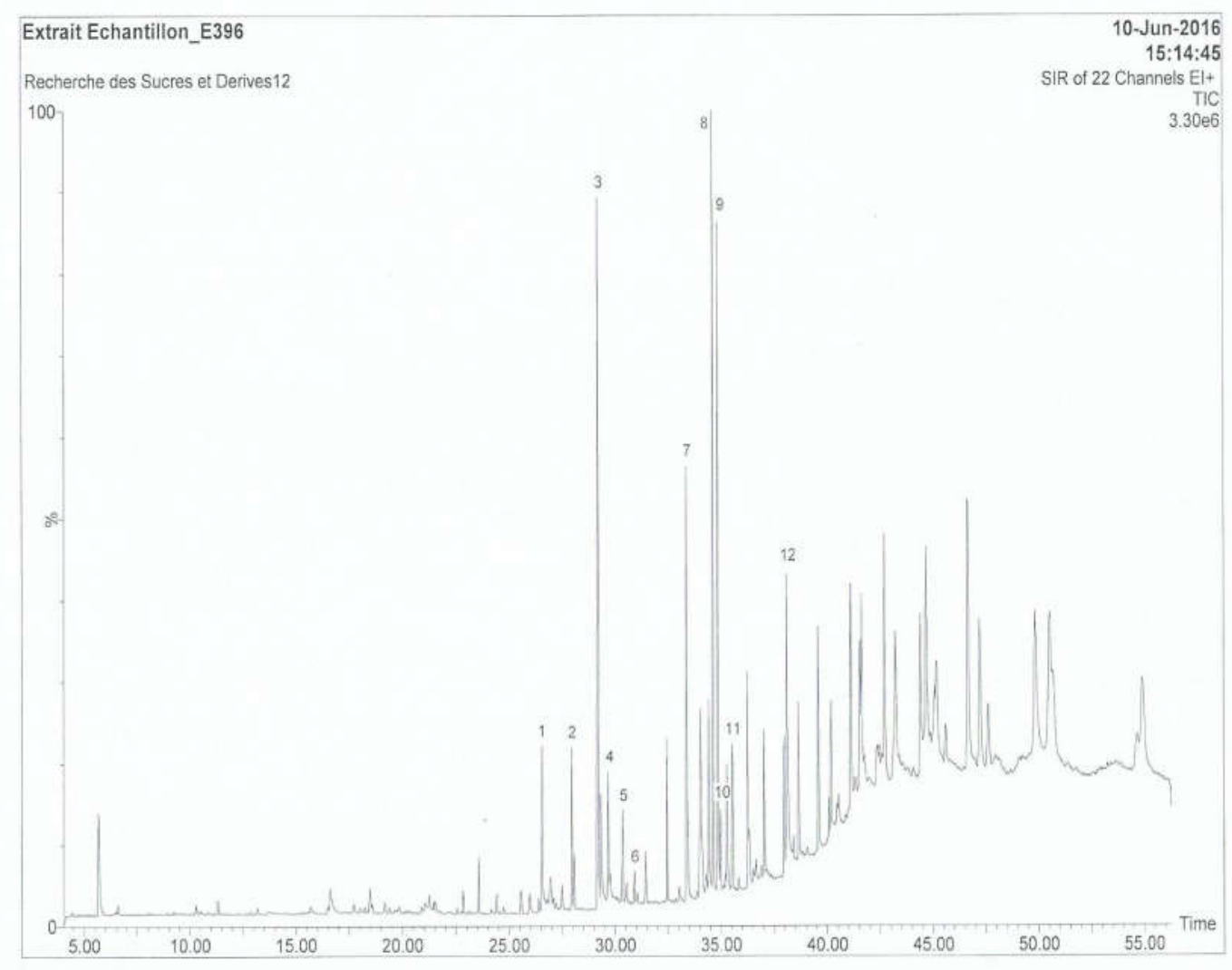

Figure 7: Chromatogramme GC-MS de cocktail à base de jus de pomme de cajou (25\%) et de jus d'ananas (75\%) (Formulation F4).

1.le Methyl 3-o-acetyl-2,4-di-o-methyl- $\alpha$-D-Xylopyranoside ; 2. le D-Glucosamine ; 3. le cis-Inositol ; 4. le Methyl (methyl 3-o-acetyl-2,4-di-o-methyl- $\alpha$-D-Mannopyranoside) urinate ; 5. le D-Glucose; 6. le D-Galactose; 7. le D-Glucohexodialdofuranoside ; 8. le 3-Tosyl sedoheptulose ; 9. le D-Threitol ; 10. le D-Ribofuranose ; 11. le $\beta$-D-Glucopyranose ; 12. le Melibiose.

Tableau 1 : Classement des différentes formulations de jus à base de pomme de cajou et d'ananas.

\begin{tabular}{l|c}
\hline Echantillons & Rang \\
\hline F4 & $1^{\text {er }}$ \\
F3 & $2^{\mathrm{e}}$ \\
F2 & $3^{\mathrm{e}}$ \\
F1 & $4^{\mathrm{e}}$ \\
\hline
\end{tabular}


Tableau 2 : Paramètres physico-chimiques des différentes formulations de jus à base de pomme de cajou et d'ananas.

\begin{tabular}{|c|c|c|c|c|}
\hline \multicolumn{5}{|c|}{ Différentes formulation à base de jus de pomme de cajou et d'ananas } \\
\hline Paramètres & F1 & F2 & F 3 & F4 \\
\hline Polyphénols totaux (mg/mL Eq Ac.Gallique) & $1,8 \pm 0,28^{\mathrm{a}}$ & $1 \pm 0,057^{b}$ & $0,96 \pm 0,06^{\mathrm{b}}$ & $0,6 \pm 0,2^{\mathrm{C}}$ \\
\hline Vitamine $\mathrm{C}(\mathrm{mg} / 100 \mathrm{~mL})$ & $317,5 \pm 18,1^{\mathrm{a}}$ & $196,61 \pm 1,46^{b}$ & $135,97 \pm 0,72^{c}$ & $52,03 \pm 1,50^{\mathrm{d}}$ \\
\hline Acidité (mol/L) & $0,02 \pm 0,1^{\mathrm{a}}$ & $0,04 \pm 0,01^{\mathrm{a}}$ & $0,06 \pm 0,01^{\mathrm{a}}$ & $0,07 \pm 0,06^{\mathrm{b}}$ \\
\hline Extraits secs réfractométrique ( ${ }^{\circ}$ brix) & $7 \pm 0,03^{\mathrm{a}}$ & $10 \pm 0,50^{b}$ & $10 \pm 0,50^{\mathrm{b}}$ & $11,5 \pm 0,01^{\mathrm{c}}$ \\
\hline $\mathrm{pH}$ & $3,76 \pm 0,22^{\mathrm{a}}$ & $3,92 \pm 0,03^{\mathrm{a}}$ & $3,53 \pm 0,06^{\mathrm{b}}$ & $4,52 \pm 0,03^{\mathrm{c}}$ \\
\hline Cendres $(\%)$ & $0,25 \pm 0,01^{\mathrm{a}}$ & $0,21 \pm 0,01^{\mathrm{a}}$ & $0,32 \pm 0,02^{b}$ & $0,41 \pm 0,04^{\mathrm{c}}$ \\
\hline Matière sèche $(\%)$ & $9,73 \pm 0,26^{a}$ & $10,5 \pm 0,50^{b}$ & $11,04 \pm 0,01 b$ & $11,83 \pm 49^{c}$ \\
\hline Lipides (\%) & $0,07 \pm 0,01^{\mathrm{a}}$ & $0,03 \pm 0,01^{\mathrm{b}}$ & $0,04 \pm 0,01^{c}$ & $0,020 \pm 0,01^{\mathrm{d}}$ \\
\hline Protéines $(\%)$ & $5,08 \pm 0,27^{a}$ & $4,21 \pm 0,10^{\mathrm{b}}$ & $4,21 \pm 0,03^{b}$ & $5,04 \pm 0,04^{a}$ \\
\hline Calcium (mg/L) & $178,43 \pm 0,01^{\mathrm{a}}$ & $220,49 \pm 0,97^{b}$ & $307,93 \pm 0,06^{\mathrm{c}}$ & $119,53 \pm 0,48^{\mathrm{d}}$ \\
\hline Potassium (mg/L) & $800,20 \pm 0,10^{\mathrm{a}}$ & $1475,57 \pm 0,40^{b}$ & $565,67 \pm 0,27^{c}$ & $941,48 \pm 0,17^{\mathrm{d}}$ \\
\hline Magnésium (mg/L) & $177,32 \pm 0,07^{\mathrm{a}}$ & $549,72 \pm 0,57^{\mathrm{b}}$ & $722,85 \pm 0,57^{\mathrm{c}}$ & $172,32 \pm 0,12^{\mathrm{d}}$ \\
\hline Fer $(\mathrm{mg} / \mathrm{L})$ & $47,16 \pm 0,31^{\mathrm{a}}$ & $100,46 \pm 0,87^{b}$ & $21,57 \pm 0,11^{\mathrm{c}}$ & $18,46 \pm 0,29^{d}$ \\
\hline Sodium (mg) & $522,3 \pm 0,12^{\mathrm{a}}$ & $286,46 \pm 0,92^{\mathrm{b}}$ & $465,04 \pm 0,09^{\mathrm{c}}$ & $479,35 \pm 0,05^{\mathrm{d}}$ \\
\hline Zinc $(\mathrm{mg} / \mathrm{L})$ & $103,38 \pm 0,21^{\mathrm{a}}$ & $37,2 \pm 0,33^{b}$ & $42,37 \pm 0,08^{\mathrm{c}}$ & $8,91 \pm 0,08^{\mathrm{d}}$ \\
\hline Fibres $(\%)$ & $0,49 \pm 0,1^{\mathrm{a}}$ & $0,52 \pm 0,03^{\mathrm{a}}$ & $0,41 \pm 0,05^{\mathrm{b}}$ & $0,32 \pm 0,02^{\mathrm{c}}$ \\
\hline
\end{tabular}

Les valeurs d'une même ligne suivies des lettres différentes sont statistiquement différentes.

$\mathrm{F} 1=100 \%$ jus de pomme de cajou

$\mathrm{F} 3=50 \%$ de jus de pomme de cajou et $50 \%$ de jus d'ananas ;

$\mathrm{F} 2=75 \%$ de jus de pomme de cajou et $25 \%$ de jus d'ananas ;

$\mathrm{F} 4=25 \%$ de jus de pomme de cajou et $75 \%$ de jus d'ananas. 


\section{DISCUSSION}

Les résultats du test hédonique par classement montrent que la formulation F4 est la préférée des dégustateurs. Ces résultats sont comparables à ceux de Carvalho et al. (2007) réalisés sur différentes formulations à base d'eau de coco et de jus de pomme de cajou. Pour cet auteur la formulation contenant $20 \%$ de pomme de cajou et $80 \%$ d'eau de coco était la préférée par les dégustateurs. Ces résultats pourraient s'expliquer par le fait que cette formulation est la plus sucrée avec un extrait sec réfractométrique de $11,5^{\circ}$ brix, supérieur à ceux des formulations F1, F2 et F3 dont les extraits secs réfractométriques varient respectivement de $7^{\circ}$ brix à $10^{\circ}$ brix. En plus la formulation $\mathrm{F} 4$ est moins astringente et à un $\mathrm{pH}$ moins acide $(4,52)$ que les autres formulations dont les $\mathrm{pH}$ varient de 3,53 à 3,92 . Cette préférence pourrait aussi s'expliquer par le fait que le jus d'ananas dominant à $75 \%$ dans ce cocktail, est un jus intégré dans les habitudes alimentaires des ivoiriens.

Les profils sensoriels des formulations montrent que la formulation F4 est de couleur jaune plus foncée, moins acide et moins astringente. Cette baisse de l'astringence pourrait s'expliquer par une diminution de la teneur en polyphénols. Cette teneur, naturellement élevée dans le jus brut de pomme de cajou (Adou et al., 2012) est responsable de l'astringence de ce dernier (Abreu et al., 2005).

La teneur en polyphénols totaux de la formulation F1 (1,8 mg/ml Eq Ac. Gallique) a subi une réduction significative après ajout de $75 \%$ du jus d'ananas jusqu'à atteindre 0,6 $\mathrm{mg} / \mathrm{ml} \mathrm{Eq} \mathrm{Ac.} \mathrm{Gallique} \mathrm{pour} \mathrm{la} \mathrm{formulation} \mathrm{F4}$ préférée par les dégustateurs. Cette faible teneur en polyphénols totaux de la formulation $\mathrm{F} 4$ serait due à la réduction de la concentration de ce jus par ajout du jus d'ananas.

Les teneurs en acide ascorbique des différentes formulations varient de 317,5 $\mathrm{mg} / 100 \mathrm{ml}$ pour la formulation $\mathrm{F} 1$ à 52,03 $\mathrm{mg} / 100 \mathrm{ml}$ pour la formulation $\mathrm{F} 4$. La teneur du jus F1 est comparable à celle obtenue par Adou et al. (2012) sur deux variétés de pommes (jaune et rouge) de Yamoussoukro dont la teneur en acide ascorbique varie de $370,9 \mathrm{mg} / 100 \mathrm{~g}$ à $480,3 \mathrm{mg} / 100 \mathrm{~g}$. Cette variation pourrait s'expliquer par le fait que la composition du fruit pourrait être influencée par divers paramètres y compris le degré de maturité (Pinheiro et al., 2006). La teneur en acide ascorbique de la formulation F4 est $52,03 \mathrm{mg} / 100 \mathrm{ml}$, valeur supérieur à celle du jus d'ananas qui est de 14,70 mg/100 ml (Akinwale, 2000). Ainsi le jus de pomme de cajou pourrait permettre d'enrichir en acide ascorbique un jus à base d'ananas.

$\mathrm{La}$ formulation $\mathrm{F} 4$ est plus riche en cendres $(0,41 \%)$ que les autres formulations dont les teneurs en cendres varient de 0,21 à $0,32 \%$. Ces cendres sont composées en partie du calcium $(119,53 \mathrm{mg} / \mathrm{l})$, suivie du potassium (941,48 mg/l), du magnésium (172,32 mg/l), du fer $(18,46 \mathrm{mg} / \mathrm{l})$, du sodium $(479,35 \mathrm{mg} / \mathrm{l})$, et du zinc $(8,91 \mathrm{mg} / \mathrm{l})$. La teneur en potassium pourrait couvrir l'apport journalier recommandé pour les nourrissons de 7 à 12 mois qui s'élève à $700 \mathrm{mg}$ (Institute of Medicine, 2001). En effet le potassium (K) participe au maintien de l'équilibre des électrolytes dans le corps humain et prévient la déminéralisation des os en empêchant la perte de calcium dans les urines (He et MacGregor, 2001). Il est un hypotenseur et intervient également dans la contraction musculaire (Dedehou, 2015). Selon Institute of Medicine (2001) la teneur en magnésium pourrait couvrir les besoins pour les enfants de 1 à 8 ans dont l'apport journalier recommandé est compris entre 65 à $110 \mathrm{mg}$. Cette teneur n'est pas négligeable pour les personnes âgées et les femmes dont l'apport recommandé varie de 200 à $350 \mathrm{mg} /$ jour. Le magnésium joue un rôle important dans la stabilité du système nerveux, dans la contraction musculaire comme un activateur de la phosphatase 
alcaline (Cowan, 2002; Ismail et al., 2011). La teneur en zinc pourrait couvrir les besoins journaliers recommandés dont la gamme varie de $2,5 \mathrm{mg} / \mathrm{l}$ pour les nourrissons à $9,4 \mathrm{mg}$ pour les hommes âgés de plus de 70 ans (Institute of Medicine, 2001). Celle en fer pourrait couvrir les besoins total des enfants jusqu'à l'adolescence dont l'apport journalier est estimé à 1 à $2 \mathrm{mg} / \mathrm{kg} /$ jour (Institute of Medicine, 2001). Le fer est un composant essentiel de l'hémoglobine qui intervient dans le transport de l'oxygène. La teneur en sodium pourrait couvrir les besoins des enfants de 0 à 12 mois dont l'apport journalier recommandé varie de 120 à $370 \mathrm{mg}$ et inférieur à celle de l'adulte variant de $300 \mathrm{mg}$ à $4700 \mathrm{mg} / \mathrm{jour}$ en fonction du sexe de l'âge et de l'état physiologique selon Institute of Medicine (2001). Le sodium $(\mathrm{Na})$ régule la pression artérielle et osmotique. C'est un cation extracellulaire important qui stabilise le fluide extracellulaire (Dedehou et al., 2015).

\section{Conclusion}

$\mathrm{La}$ formulation des différentes boissons non alcoolisées à base de pomme de cajou et d'ananas est une voie de valorisation de la pomme de cajou. Ces formulations montrent que le jus brut de cajou, riche en éléments nutritifs et en vitamine $\mathrm{C}$ peut être utilisé pour améliorer la teneur en micronutriments (vitamines et minéraux) d'autres produits pauvres en ces différents éléments comme l'ananas, la mangue, etc...

Dans le cas de l'enrichissement de l'ananas il en ressort plusieurs cocktails parmi lesquels la formulation F4 (mélange de 25\% de jus de pomme de cajou et $75 \%$ de jus d'ananas), à des caractéristiques physicochimiques et sensorielles préférées par les dégustateurs. Cet enrichissement donne naissance à un nouveau produit riche en minéraux et bénéfique pour les consommateurs. Les minéraux sont des micronutriments qui jouent un rôle important dans le fonctionnement de l'organisme. La consommation de ce cocktail à base du jus de pomme de cajou et d'ananas apporte à l'organisme plusieurs minéraux pour son bon fonctionnement. Du point de vue sensoriel, l'ananas par son arôme et son goût a amélioré l'appréciation globale des dégustateurs. Cette étude a permis aussi de réduire l'astringence prononcée du jus brut de pomme de cajou. Ainsi ces résultats mettent à la disposition des transformateurs des informations utiles pour la production de cocktails à base de jus de pomme de cajou et d'ananas.

\section{CONTRIBUTIONS DES AUTEURS}

GSO a réalisé les travaux. Il a exploité les résultats et rédigé le manuscrit. DS a participé à l'acquisition de la matière première et à la réalisation des manipulations au laboratoire. KOC a participé à l'amélioration $\mathrm{du}$ manuscrit par sa correction. EKK a contribué à l'amélioration du manuscrit, validé les protocoles de laboratoire et supervisé tous les travaux. Il a été le responsable scientifique de cette étude.

\section{CONFLIT D'INTERETS}

Les auteurs de ce manuscrit déclarent ne pas exister de conflit d'intérêt.

\section{REFERENCES}

Aboh André Boya, Dougnon Jacques T, Théodora Atchade, Ghislaine S, Tandjiekpon André M. 2011. Effet d'aliments à base de pomme cajou sur les performances pondérale et la carcasse des canetons en croissance au Bénin. Int. J. Biol. Chem. Sci., 5(6): 2407-2414.

Abreu Fernando AMP, Dornier M, Reynes M. 2005. Potentialités de la microfiltration tangentielle sur membranes minérales pour la clarification du jus de pomme de cajou. Fruits. 60: 33-40.

Adou M, Kouassi DA, Tetchi FA and Amani NG. 2012. Phenolic profile of Cashew (Anacardium ocidentale L.) of Yamoussoukro and Korhogo, Côte 
d'Ivoire. Journal of Applied Biosciences, 49: 3331-3338.

Akinwale TO. 2000. Cashew apple juice: Its use in fortifying the nutritional quality of some tropical fruits. Eur. Food Res. Technol., 211: 205-207.

AOAC. 1984. Official methods of analysis of the Association of Official Analytical Chemists (14th edn) Washington, DC.

AOAC (Association of Official Analytical Chemists). 1990. The Official Methods of Analysis (15th edn). Washington, DC, 1230p.

AOAC. 1995. Official methods of Analysis of the Association of Official Analytical Chemists Chemists International, $\left(16^{\text {th }}\right.$ edn). AOAC International Arlington, VA, $250 \mathrm{p}$.

Assunção RB, AZ Mercadante. 2003. Carotenoids and ascorbic acid composition from commercial products of cashew apple (Anacardium occidentale L.). Journal of Food Composition and Analysis, 16(6): 647-657.

Assunção RB, Mercadante AZ. 2003. Carotenoids and ascorbic acid from cashew apple (Anacardium occidentale L.): variety and geographic effects. Food Chemistry, 81(4): 495-502.

CCA (Conseil Coton Anacarde en Côte d'Ivoire). 2016. Publié le lundi 15 février.

Cowan JA. 2002. Structural and catalytic chemistry of magnesium-dependent enzymes. BioMetals, 15: 225-235.

Dedehou ESCA, Dossou J, Soumanou MM. 2015. Etude diagnostique des technologies de transformation de la pomme de cajou en jus au Bénin. Int. J. Biol. Chem. Sci., 9(1): 371-387.

Diez M, Nguyen P. 2006. Obésité : épidémiologie, physiopathologie et prise en charge $\mathrm{du}$ chien obèse. In Encyclopedie de la Nutrition Clinique Canine, Pibot P, Biourge V, Elliot D (eds). Aniwa: Paris; 2-57.
FAO (Food and Agriculture Organization). 2014. Base des données de la FAO 2011. http://faostat3.fao.org. Visité le 4 Septembre 2014 à 13 heures.

Gouma M. 2003. Analyse des négociations de l'OMC sur l'agriculture pour la formation d'une politique appropriée au développement de la filière anacarde en Côte d'Ivoire, Mémoire professionnel, Université de Cocody, Abidjan, 46 p.

He FJ, MacGregor GA. 2001. Beneficial effects of potassium. Br. Med. J., 323: 497-501.

Institute of Medicine. 2001. Food and Nutrition Board, National Academies.

IRD (Institut de Recherche pour le Développement). 2012. The cashew cookbook: Recipes from the Gambia and Senegal, IRD, 42p.

Lautié E, Dornier M, de Souza FM, Reynes M. 2001. Les produits de l'anacardier: caractéristiques, voies de valorisation et marchés. Fruits, 56: 235-248.

Martin PJ, Topper CP, Bashiru RA, Boma F, De Waal D, Harries HC, Kasuga LJ, Katanila N, Kikoka LP, Lamboll R, Maddison AC, Majule AE, Masawe PA, Millanzi KJ, Nathaniels NQ, Shomari SH, Sijaona ME, Stathers T. 1997. Cashew nut production in Tanzania: Constraints and progress through integrated crop management. Crop Protection, 16(1): 514.

Meilgaard, Civille GV, BT. 1999. Carr Sensory Evaluation Techniques (3rd Edn). CRC Press: New York.

Michodjehoun-Mestres L, Souquet J-M, Fulcrand $\mathrm{H}$, Bouchut $\mathrm{C}$, Reynes $\mathrm{M}$ et Brillouet J-M. 2009. Monomeric phenols of cashew apple (Anacardium occidentale L.). Food Chemistry, 112: 851-857.

Michodjehoun-Mestres L, Souquet JM, Fulcrand H, Meudec E, Reynes M, Brillouet JM. 2009. Characterisation of highly polymerised prodelphinidins from skin and flesh of four cashew apple 
(Anacardium occidentale L.) genotypes. Food Chemistry, 114: 989-995.

Pinheiro AM, Fernandes AG, Cavalente AEF, Do Prado GM, De Sousa PHM, Maia GA. 2006. Avaliação química, físico-quimica e microbiológica de sucos de frutas integrais: Abacaxi, caju e Maracujá. Ciênc. Tecnol. Aliment, Campinas, 26(1): 98-103.

Rey JY. 1998. La noix de cajou en Côte d'Ivoire en forte progression. Fruitrop., 51: 12-13.

Sekhar GS, Rajkeshwar P, Anil P. 2013. In Silico Analysis of Stem Bromelain. International Journal of Pharmaceutical and Chemical Sciences, 2(2): 992-997.

Soro D. 2008. Concentration par microfiltration tangentielle et caractérisation d'extraits carotenoïdiques de pomme de cajou. Institut des régions chaudes. Master Recherche Naval, Montpellier, SupAgro, 67 pages.

Trevian MTS, Pfundstein B, Haubner R, Würtele G, Spiegelhalder B, Bartsch H, Owen RW. 2005. Caracterisation of alkyl phenols in cashew (Anacardium occidentale L.) products and assay of their antioxidant capacity. Food and Chemical Toxicology, 44: 188-197.

Vinson JA, Yong A, Xuelci S, Ligid Z, Bose P. 2001. Phenol antioxidant and quantity and quality in foods. J Agric Food Chem., 49: 5315-5322.

Wood JE, Senthilmohan ST, Peskin AV. 2002. Antioxydant activity of prodyanidin-containing plant extracts at different pHs. Food Chem., 77: 155-161. 